\title{
Compromiso Meníngeo en Leucemia Aguda del Niño
}

\author{
Dra. Paola Zolezzi R. 1-2; T.M. Mariana Araneda Z. ${ }^{3}$; Dra. Cecilia Faúndez V.4; \\ Dra. Alexis Strickler P.4; Enf. U. Patricia Niada G. ${ }^{5}$; Dr. Claudio Zapata 0.2 .
}

\section{Meningeal Leukemia in Children}

\begin{abstract}
Among 56 children with acu te leukemia (39 ALL and 17 AML), 13 (23\% 8 ALL and 5 AML) showed meningeal compromise. Eight had two or more meningeal leukemia predictive factors with increased leukemic blast cells and associated thrombocy topenia. The meningeal leukemia group had a high mortadity $(88.8 \%$ ALL and $75 \%$ AML) which was telated to the cytologic type (only one patient with $A L^{1}$ type), a higher frequency of meningeal compromise at the time of the initial diagnosis of leukemia, and age below two and above ten years old. The two patients remaining alive are in complete remission with survivals of 75 and 96 months respectively. At present, the treatment of meningeal leukemia is subject of controyersy. The Chileas Pediatric Oncology Group (GOPECH) has recommended a therapeutic schedule that inciudes the different criterias that are prevalent in current literature.
\end{abstract}

La infiltración leucémica de las meninges constituye un serio problema en el manejo de la Leucemia Aguda (LA) Infantil' .

El diagnóstico de Leucemia Meníngea (LMe) se establece frente a la presencia de células blásticas en el líquido céfalorraquídeo (LCR) y/o la demostración de un número aumentado de células mononucleares (más de $10 \times \mathrm{mm}^{3}$ ) en el LCR $^{2}$.

La LMe puede presentarse en cualquier etapa

Médico. Instituto de Pediatría. Universidad Austral. Médico, Instituto de Hematologia, Universidad Austral.

3 Tecnólogo Médico, Instituto de Histología y Patología. Universidad Austral.

4 Médico. Servicio de Pediatr ja, Hospital de Valdivia.

5 Enf. Universitaria, Servicio de Pediatria, Hospital de Valdivia. de la enfermedad leucémica: inicialmente o durante la remisión, precediendo en meses o acompañando una recidiva medular ${ }^{3}$. La comprobación de compromiso infiltrativo meningeo una vez lograda la remisión completa (RC) en una leucemia aguda, reduce la posibilidad de lograr una curación total, y disminuye la sobrevida ${ }^{4}$. Esto ha llevado a incluir la profilaxis del sistema nervioso central (SNC) con radioterapia (RDT) craneal, quimioterapia (QMT) intratecal (i.t.) o ambas como parte inicial del tratamiento de la leucemia aguda linfática (LAL) ${ }^{5-6}$. Con esta medida se ha reducido la incidencia de LMe como primera evidencia de recidiva extramedular en la LAL del niño de $50 \%$ a $5 \%$.

Factores predictivos en el momento del diagnóstico inicial de LA, como recuento leucocitario superior a $10.000 \mathrm{x} \mathrm{mm^{3 }}$ con alto porcentaje de 
blastos, recuento plaquetario interior a $50.000 \mathrm{x}$ $\mathrm{mm}^{3}$, adenopatías iniciales prominentes y edad inferior a un año, han permitido identificar pacientes con alto riesgo de presentar una LMe, recomendándose en ellos agregar profilaxis mantenida con QMT i.t. ${ }^{2 * 4}$.

Pese a esto, la LMe constituye la forma más frecuente de recidiva extramedular y el tratamiento de esta complicación sigue siendo un desafio, pese a los numerosos ensayos clínicos realizados ${ }^{7-8-9}$.

Se revisa nuestra experiencia en un grupo de menores de 15 años, portadores de síndrome leucémico agudo linfático y mieloide, que desarrollaron L Me.

\section{MATERIAL Y METODO}

Se analizan 13 pacientes con LMe de un total de 56 menores de 15 ar̃os con LA provenientes de la Décima Región, ingresados en el periodo 1976-1981 en los Servicios de Pediatria, Oncologia y Radioterapia del Hospital Regional de Valdivia e Instituto de Hematología de la Facul. tad de Medicina de la Universidad Austral de Chile. Los resultados fueron evaluados en Junio-84.

E1 diagnóstico de LA se confirmó con mielograma (tinción de May-Grünwald-Giemsa) estableciéndose el tipo citológico de acuerdo a la clasificación $\mathrm{FAB}^{10}$, complementado con tinciones citoquímicas (PAS, mieloperoxidasas, lípidos) ${ }^{11}$.

El tratamiento QMT, tanto para la leucemia aguda linfática (LAL) como mieloide (LAM), consistió en: Una fase de inducción según el esquema $\operatorname{COAP}^{12}$, que incluyó Ciclofosfamida $100 \mathrm{mg} / \mathrm{m}^{2}$ días 1 al 5 , oral; Vincristina $1 \mathrm{mg}$ día 1, i.v.; Citosina-arabinósida (Ara-C) $100 \mathrm{mg} / \mathrm{m}^{2}$ días 1 al 5, i.v. y Prednisona 50-100 mg dias 1 al 5 , oral, seguidos por 9 dias de descanso después de los cuales se repiten las curas hasta obtener la $\mathrm{RC}$, de acuerdo al criterio de Lampkin ${ }^{5}$.

Desde el año 1979, se ha agregado metotrexato (MTX) i.t. $6,25 \mathrm{mg} / \mathrm{m}^{2}$ por una vez, durante la fase de inducción el día 1 de la primera cura.

La profilaxis del $\mathrm{SNC}^{13}$ se realizó con RDT craneal $2.400 \mathrm{rads}$ y MTX i.t.: $12 \mathrm{mg} / \mathrm{m}^{2}$, administrado c/3 días, por 5 veces.

En la fase de mantención se aplicó el esquema POMP ${ }^{14}$, modificado, usando 6 Mercaptopurina $300-500 \mathrm{mg} / \mathrm{m}^{2}$, días 1 al 5 , oral; Vincristina $1 \mathrm{mg}$ día 1, i.v.; Metotrexato $5-7,5 \mathrm{mg} / \mathrm{m}^{2}$ días 1 al 5, oral, seguidos por 9 días de descanso a cuyo término se repiten las curas durante $\mathbf{3 0}$ meses, corrigiéndose las dosis según la tolerancia individual.
Se administró MTX i.t.: $12 \mathrm{mg} / \mathrm{m}^{2}$, cada 12 semanas en los pacientes con factores predictivos de desarrollar una LMe a partir del año 1979.

Se estableció al diagnóstico de LMe cuando habian sintomàs y signos sugerentes de hipertensión intracraneana como vómitos, cefalea, letar. go, edema de papila, signos meningeos, convulsiones $^{15}$, o células blásticas en el $L C R \circ$ un número aumentado de células mononucleares (más de $10 \times \mathrm{mm}^{3}$ ) en este último ${ }^{6 \cdot 16}$.

El tratamiento de la LMe consistió en la administración de MTX i.t.: $12 \mathrm{mg} / \mathrm{m}^{2}$, una a dos veces por semana, hasta obtener dos LCR con recuento celular normal $y$ sin células blásticas, seguido de RDT craneal con 2.000 a 2.400 rads. Se continuó con MTX i.t. de mantención cada 12 semantas hasta finalizar el tratamiento sistémi$\mathrm{CO}^{4 \cdot 17}$.

Cuando se sospechó toxicidad secundaria a la quimioterapia j.t. y/o la RDT, se agregó hidrocortisona (HC) i.t. $30 \mathrm{mg} / \mathrm{m}^{2}$.

Se hicieron estudios de LCR, incluyendo búsqueda de blastos, recuento celular, químico y cultivo, al ingreso y cada vez que se realizó QMT i.t.

\section{RESULTADOS}

La incidencia de LMe en el grupo estudiado corresponde al $23,2 \%$. En relación al tipo citoló. gico, esta incidencia es similar: 9 de 39 LAL $(23,1 \%)$ y 4 de 17 LAM $(23,5 \%)$.

El análisis de los factores predictivos de riesgo de LMe en el momento del diagnóstico inicjal de LA (Figura 1), mostró que 8 de los 13 pacientes tenian dos o más factores predictivos de $\mathrm{LMe}$. Sólo un caso correspondia a LAL de riesgo común (tipo $L_{1}$ ), ocho pacientes presentaban características citológicas de LAL de alto riesgo y cuatro casos correspondían a LAM.

El conjunto de síntomas y signos sugerentes de $\mathrm{LMe}$ fue poco notorio en cinco pacientes, encontrándose cefalea, vómitos, irritabilidad y letargo en los ocho restantes, dos de los cuales desarrollaron además compromiso de pares craneanos (VII par).

En el LCR se encontraron-blastos en 12 de los 13 pacientes, con pleiocitosis y descenso de la glucosa en 10. En un solo paciente hubo pleiocitosis sin blastos, con leve aumento de las proteínas. Los cultivos bacteriológicos fueron negativos en todas las muestras.

Con respecto al momento de presentación de la LMe, se pueden separar dos grupos (Figura 2): El primero corresponde a 8 pacientes con LMe en el momento de] diagnóstico inicial (Fase de Inducción), 4 LAL y 4 LAM. En esta etapa, conjuntamente con la QMT sistémica destinada a 
Tabla 1.

Tipo citológico y factores predictivos de Leucemia Meníngea

\begin{tabular}{|c|c|c|c|c|c|}
\hline$C A W O$ & $\begin{array}{l}\text { FDAD } \\
\text { AÑUS' }\end{array}$ & $\begin{array}{c}\operatorname{lIPO} \\
\text { CIISU (K. }\end{array}$ & 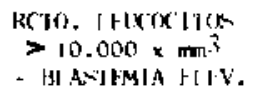 & $\begin{array}{l}\text { KCIO. PI.A(H'FIAL } \\
<50.000 \times \mathrm{mm}^{3}\end{array}$ & 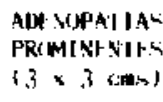 \\
\hline 1 & 14 & $\mathrm{I}_{2}$ & + & - & - \\
\hline 2 & 10 & $M_{2}$ & + & + & + \\
\hline 3 & $x$ & $M_{2}$ & - & 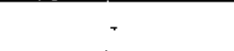 & - \\
\hline 4 & 1 & 1.2 & + & . & - \\
\hline 5 & 2 & $M_{1}$ & - & - & - \\
\hline 6 & 10 & $\mathrm{I}_{2}$ & . & - & + \\
\hline 7 & 12 & 1.2 & + & - & + \\
\hline$\dot{s}$ & 7 & $M_{1}$ & - & - & - \\
\hline (3) & 4 & 1.1 & + & + & - \\
\hline 10 & 6 & $\mathrm{I}_{2} 2$ & 4 & + & - \\
\hline 11 & 4 & 1.3 & + & + & - \\
\hline 12 & 2 & 1.2 & - & - & - \\
\hline 1.3 & 2 & $\mathrm{I}_{2}$ & + & + & + \\
\hline
\end{tabular}

Tabla 2.

Resumen de trece pacientes con Leucemia Meníngea

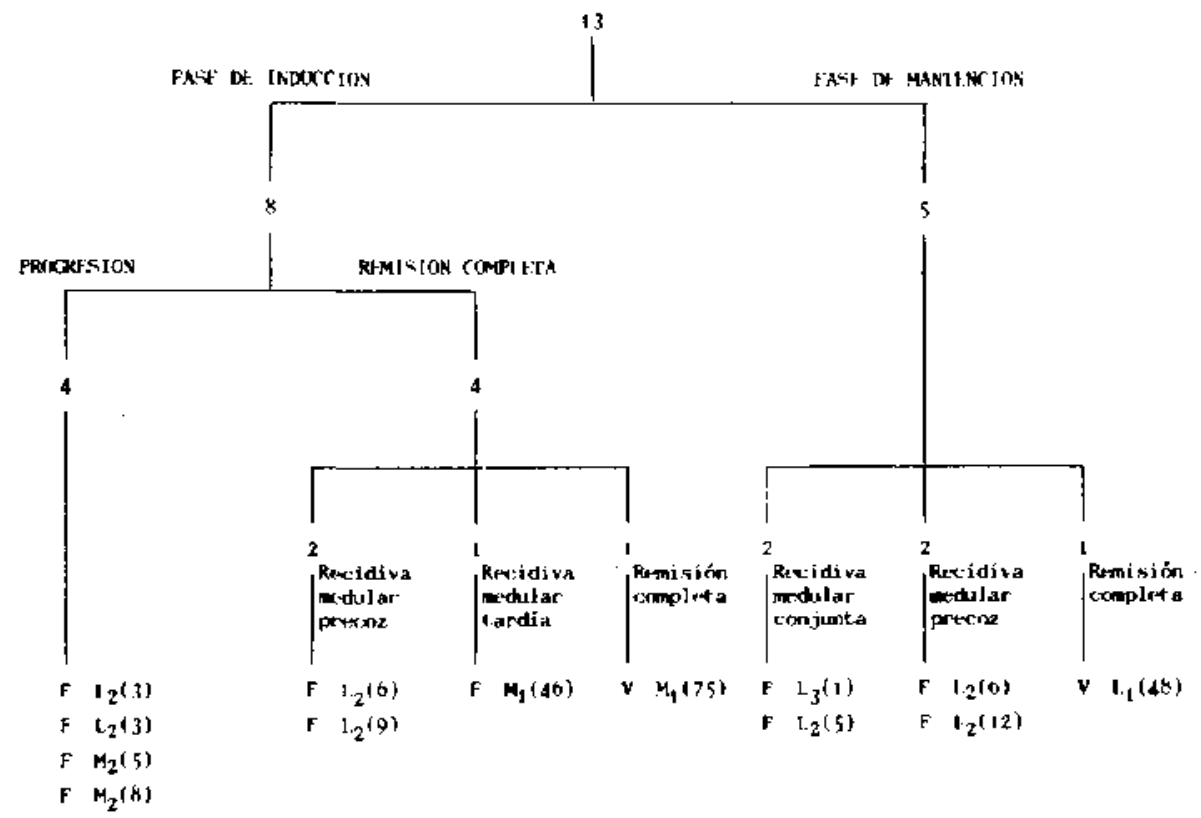


lograr la remisión medular, los ocho recibieron trạtamiento para su LMe. En cuatro se observó progresión de su leucemia con intensificación de los síntomas y signos meningeos, comprone. tiéndose el VII par craneal en dos de ellos. Todos fallecieron entre $\operatorname{los} 3$ y 8 meses de diagnosticada la LMe. En los otros cuatro se obtuvo RC, desapareciendo la infiltración meníngea. En dos, la remisión fue de corta duración, pues fallecie. ron de recidiva medular, respectivamente 6 y 9 meses después de haber presentado la LMe. E1 tercer paciente, después de 44 meses de RC, presentó una recidiva medular y falleció dos meses más tarde. El cuarto paciente, permanece en RC 75 meses, habiéndose suspendido toda quimioterapia sistémica e j.t. de mantención a los 36 meses de tratamiento.

E] segundo reune a los 5 pacientes que desarrollaron LMe durante la evolución (Fase de Mantención) entre los 40 que lograron RC ( 30 LAL y 10 LAM). Todos eran LAL, presentaron la LMe durante la fase de mantención (con una mediana de aparición de 12 meses) y habian recibido profilaxis del SNC con RDT craneal (2.400 rads) y MTX i.t. (12 mg/m $/ \mathrm{m}^{2}$ por 5 veces). Dos, después de 17 y 12 meses de RC, presen. taron conjuntamente infiltración meníngea y me. dular, falleciendo 1 y 5 meses después respectivamente. Dos pacientes tuvieron LMe después de 6 y 5 meses de RC, seguida de recidiva medular a los 4 y 10 meses respectivamente, falleciendo ambos dos meses más tarde. El quinto sujeto sufrió compromiso meníngéo después de $35 \mathrm{me}$. ses de RC, recibiendo tratamiento para LMe, seguido de prolongación de la QMT sistémica e i.t. de mantención por dos años más, conpletando 96 meses de sobrevida to tal.

\section{DISCUSION}

Por ser la leucemia una enfermedad sistémica, puede manifestar algún grado de compromiso del $\mathrm{SNC}^{18}$ en cualquier etapa de su evolución. En nuestro grupo de LAL ( 39 pacientes), la incidencia de LMe en el nomento del diagnóstico inicial fue $10,2 \%$ ( 4 de 39), cifra superior al $5 \%$ comu. nicada por Miller ${ }^{19}$, quién establece la necesidad de separar a los nifios que presentan compromiso inicial del SNC por constituir un subgrupo de mal pronóstico que necesitará un tratamiento más agresivo.

Una vez obtenida la $\mathrm{RC}$ y habiéndose realizado una profilaxis adecuada del SNC, el compromiso meníngeo se manifestó en el $16,6 \%$ ( 5 de 30 LAL que entran en RC), Hustu ${ }^{7}$ comunica $14 \%$ de compromiso del SNC en LAL en RC habiendo recibido tratamiento profilactico del SNC (irradiación craneal y MTX i.t.), mientras que en el medio nacional, Mardones ${ }^{20}$ refiere $15 \%$. La mediana de presentación de 12 meses es similar a la comunicada recientemente en nuestro medio por Advis 21 .

La recidiva por compromiso meníngeo fue seguida por recidiva medular en cuatro de los cinco pacientes, situación que ocurrió hasta en el $60 \%$ de los casos de Robinson ${ }^{22}$ y Simone ${ }^{23}$.

La mortalidad de $88,8 \%$ ( 8 de 9 LAL) está dada, a nuestro parecer, tanto por la mayor incidencia de LMe en el momento del diagnóstico de LA $^{19}$ como por el tipo citológico $\mathrm{L}_{2}$, habiendo informado $\mathrm{Kay}^{24}$ en este grupo citológico una incidencia de recidiva tres veces mayor. Estos dos hechos, sumados a la presentación en menores de dos años y mayores de diez son los tres factores más negativos de este grupo de pacientes, $y$ han sido destacados últimamente por Miller ${ }^{25}$ en un análisis de 880 pacientes con LAL, Kay ${ }^{24}$ y Komp ${ }^{26}$. De nuestros pacientes en esta situacjón sólo uno se encuentra vivo con sobrevida desde el diagnóstico inicial de leucemia de 96 meses, correspondiendo al único con tipo citológico $\mathrm{L}_{1}$.

En nuestro grupo de LAM la incidencia de LMe es de $23,3 \%$ (4 de 17 casos), siendo llamativo que todos los casos la presentaron desde el momento del diagnóstico inicial. Esta incidencia es algo superior al $14 \%$ comunicado por Dahl' ${ }^{27}$ y por Choi ${ }^{28}$. Para Dahl ${ }^{27}$, la LMe en el momento del diagnóstico de LA es más frecuente en las formas mieloides. No obstante, obtenida la RC, la aparición de LMe como sitio inicial de recidiva es menor en LAM quf en $\mathrm{LAL}^{27.29}$, estimándose que alcanza un $10 \mathrm{a}$ un $15 \%$ de los casos, aunque para Madanat ${ }^{30}$, la incidencia real se desconoce por cuanto no se exanina en forma rutinaria el LCR en pacientes con LAM.

El pronóstico de esta complicación es ominoso, lo que se relaciona en nuestro grupo con una mortalidad de $75 \%$ ( 3 de 4 pacientes) y con un paciente vivo en $\mathrm{RC}$ con una sobrevida de 75 meses.

Los síntomas y signos de compromiso del SNC son inespecificos ${ }^{31}$; no existiendo diferencias en cuanto al tipo de kucemia ${ }^{32}$. Cuando están presentes, como en 8 de nuestros pacientes, son generalmente expresión de la hipertensión intracraneana que debe orientar al diagnóstico de compromiso leucémico del SNC, aún en ausencia de alteraciones del $\mathrm{LCR}^{31-3.3}$

La identificación de células blásticas en el LCR no ofreció dificultades, habiéndose tomado las muestras antes de inyectar el MTX i.t., lo que tiene importancia pues éste puede provocar plejocitosis 34-35.

El tratamiento de la LMe es muy controver. 
tido, ya que algunos autores recomiendan el uso de RDT craneal o cráneo-espinal, otros prefieren sólo QMT i.t. y algunos, asociación de QMT i.t. y RDT. Asi, Miller ${ }^{19}$ recomienda en la LAL, RDT craneal (1.800 rads) más RDT espinal (1.000 rads), si no se ha usado previamente MTX a altas dosis. Sullivan ${ }^{2}$, plantea tanto para LAL como LAM el uso intratecal de: MTX $15 \mathrm{mg} / \mathrm{m}^{2}$, Ara-C $30 \mathrm{mg} / \mathrm{m}^{2}$ e HC $15 \mathrm{mg} / \mathrm{m}^{2}$, cada 4 dias hasta que los mononucleares en el LCR disminuyan à menos de 10 células $x \mathrm{~mm}^{3}$ y no se encuentren blastos, seguido de mantención con MTX i.t. cada 8 semanas, con lo que obtiene prolongaciones de la remisión ${ }^{17}$. Mauer ${ }^{36}$ recomienda para la LAL la administración de MTX i.t. $12 \mathrm{mg} / \mathrm{m}^{2}$ semanal por 6 veces; si aún hay blastos en el LCR agrega dosis adicionales hasta obtener dos LCR libres de células leucémicas, sin sobrepasar un total de 12 dosis; si hay pleiocitosis progresiva o aumento de la concentración de proteínas en el LCR, suspende el uso de MTX i.t. y administra RDT cráneo-espinal en dosis de 1.500 a 2.400 rads en dos a seis semanas, dependiendo de factores tales como edad del niño, presencia de signos neurológicos focales y tolerancia individua; si hay resistencia al MTX i.t. y fracaso a la RDT posterior, recomienda el uso de Ara-C i.t. entre 5 a $70 \mathrm{mg} / \mathrm{m}^{2}$ y dada la tendencia a recidivar de LMe, administra MTX i.t. $12 \mathrm{mg} / \mathrm{m}^{2}$ cada 6 semanas durante la manten. ción. En un esquema más sencillo Foon ${ }^{37}$, utiliza RDT craneal con 2.400 rads administrados en 12 fracciones en tres semanas más Ara-C i.t. 20-50 $\mathrm{mg} / \mathrm{m}^{2}$ cada tres a cinco días hasta que las células desaparezcan del LCR, pudiendo administrarse dosis más altas de Ara-C i.t. de $100 \mathrm{mg} / \mathrm{m}^{2}$, dosis que pueden producir neurotoxicidad en algunos pacientes ${ }^{38-39}$. Hagbin ${ }^{40}$, utilizando tratamientos más intensivos con QMT i.t. (MTX y Ara-C) más irradiación del neuroeje (600 rads) y administración de QMT intraventricular de MTX mediante el reservorio de Ommaya, cada 8 semanas durante 3 años, obtiene prolongaciones importantes de la remisión.

En nuestro medio, el Grupo Oncológico Pediátrico Chileno GOPECH ${ }^{4}$ reune los criterios anteriormente expuestos al proponer el uso de QMT i.t. con tres drogas (MTX $15 \mathrm{mg} / \mathrm{m}^{2}+$ Ara-C $60 \mathrm{mg} / \mathrm{m}^{2}+\mathrm{HC} 30 \mathrm{mg} / \mathrm{m}^{2}$ ) dos veces por semana, hasta obtener la desaparición de los blastos en el LCR, con un mínimo de 5 dosis; a continuación de las punciones lumbares, RDT craneal (2.500 rads) y espinal (1.000 rads), reiniciando conjuntamente el tratamiento sistémico.

El tratamiento en nuestros pacientes consistió en el tso de RDT craneal y MTX j.t., con adición de $\mathrm{HC}$ i.t. ante efectos colaterales. Uno de nuestros pacientes presentó una leucoencefalopatía por MTX i.t. y/o RDT $42-43-44$, y pese al uso de leucovorina ${ }^{4-46}$, el cuadro progresó falleciendo el paciente doce meses después. Desde 1982, hemos adoptado el esquema de tratamiento propuesto por GOPECH.

El desarrollo de técnicas complementarjas de diagnóstico en lMe permitirá un tratamiento más oportuno, como lo ha demostrado la determinación por inmunofluorescencia indirecta de la deoxinucleotidil transferasa terminal en LCR de

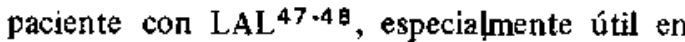
aquellos casos con blastos de morfología atípica y bajos recuentos en el LCR.

Sin embargo, la única arma eficaz de prevención para la LMe es la profilaxis del SNC, tanto en LAL como en LAM con alguno de los diferen. tes esquemas de profilaxis en uso $49 ; 50.51$, pese a que estos tratamientos pueden asociarse con secuelas adversas con el corret del tiempo 2-5 3 .

\section{RESUMEN}

En un grupo de 56 niños con leucemia aguda (39 LAL y 17 LAM), 13 (23\%: 8 LAL y 5 LAM) presentaron compromiso meníngeo. Ocho pacientes tenían dos o más factores predictivos de desarrollar una LMe, destacando las blastemias elevadas y la trombocitopenia asociada. El grupo con LMe tuvo una elevada mortalidad $(88,8 \%$ en LAL y $75 \%$ en LAM), atribuída al tipo citológico (sólo un paciente perteneciente al tipo $\mathrm{LAL}_{1}$ ), a la mayor frecuencia de compromiso meningeo en el momento del diagnóstico inicial de leucemia y a las edades bajo dos y sobre diez años. Los dos pacientes vivos están en $\mathrm{RC}$ con una sobrevida de 75 y 96 meses.

E] tratamiento de la leucemia meníngea es muy controvertido. El Grupo Oncológico Pediátrico Chileno (GOPECH) ha recomendado un esquema terapéutico que reune ctiterios diferentes.

\section{AGRADECIMIENTOS:}

A los Médicos e Internos de la Unidad de Medicina Infantil del Servicio de Pediatría del Hospital de Valdivìa.

\section{REFERENCIAS}

I Aur RJ, Simone JV. Hustu HO, Verzosa MS: A comparative study of central nervous system irradiation and intensive chemotherapy eariy in remission of childhood acute lymphocytic leukemia. Cancer 29: $381,1972$.

2 Sullipan MP. Hrgovcic M.: Extramedullary leukemia. In: Sutow WW, Vietti TJ, Fernbach DJ (eds): “Clinical Pediatric Oncology", Saint Louis $\mathrm{MO}, \mathrm{CV}$ Mosby, 1977, pp 371-396.

3 Pochedily C.: Neurologic manifestations in acute leukemia. I. Symptoms due to increased cerebros- 
pinal fluid pressure and hemorrhage. NY State J. Med. 575, 1975.

4 Sullivan MP: Prognosis in central nervous system leukemia. Seventh National Cancer Conference Proceedings, 1973, pp 593-599.

s Lampkin BC, ACWilliams NB, Mauer AM: Treatment of acute leukemia. Pediatr. Ciin. North Am. 19: $1123,1972$.

- Haghbin M. Tan ChTC, Clarkson BD, Miké V., Burchenal JH, Murphy ML: Treatment of acute lymphoblastic leukemia in children with "prophy. lactic" intrathecal methotrexate and intensive systemic chemotherapy. Cancer Res. 35: 807, 1975.

7 Hustu $O H$, Aur RJ: Extramedullary leukema. Clin. Heematol 7: 313-337, 1978.

B Sullivan MP, Humphrey GB, Vietti TJ, Haggard ME, Lee E: Superiority of conventional intrathecal methotrexate therapy with maintenance over intensive intrathecal methotrexate therapy, unmaintained, or radiothesapy $(2.000-2.500$ rads tumor dose) in treatment for meningeal leukemia. Cancer 35: 1066 , 1975.

9 Willoughby ML: Treatment of overt meningeal leukemia in children: results of second MRC meningeal leukemia trial. Brit. Med. J. 1: 864, 1976.

10 Bennett $M$, Catovsky $D$, Daniel MT, Flandrin $G$, Galton DA. Gratnick HR, Sultan C.: Proposals for the classification of the acute leukaemias. FrenchAmerican-British (FAB) Co-operative Group. Br. J. Haematol. 33; 451, 1976 .

11 Hayhoe $F G$, Cawley JC- Acute leukemia: cellular morphology, cytochemistry and fire structure. Clin. Haematal. 1: 49, 1972.

12 Bodey GP, Rodriguez $V_{*}$, Whitecar $I P$, Hort $J$. Freireich EJ: The treatment of acute leukemia in adutts. In "Leukemia-Lymphoma". The Liniversity of Texas, MD Anderson Hospital and Tumor Institute, Houston TX. Chicago, Year Book Medical Publishers Inc., 1970, pp 333-345.

13 Aur RJ, Hustu OH, Verzosa MS, Wood A., Simone $J V$ : Comparison of two methods of preventing central nervous system leukemia. Blood 42: $349,1973$.

14 Rodríguez $V$, Hart JS, Freireich EJ, Bodey GP. McCredie KB, Whitecar JP, Coltman CA: POMP combination chemotherapy of adult acute leukemia. Cancer 32: 69, 1973.

15 Wolff JA: Acute leukemia in children. Clin. Haematol. 1: 189-226, 1972.

16 Aaronson AG. Hadiu SI, Melamed MR: Spinal fluid cytology during chemotherapy of leukemia of the central nervous system in children. Am. J. Clin. Pathol. 63: 528, 1975.

17 Sullivan MP, Vietti TJ, Haggard ME, Donaldson MH, Krall $J M$, Gehar EA: Remission maintenance therapy for meningeal leukemia: intrathecal methotrexate vs intravenous bis-nitrosurea. Blood 38 : $680,1971$.

18 Price RA, Johnson WW: The central nervous system in childhood leukemia: I.,The arachnoid. Cancer 31 : $520,1973$.

19 Miller $D R$ : Acute lymphoblastic leukemia. Pediatr. Clin. North Am. 27: 269, 1980.

20 Mardones P., Marcheysky S., Walter T., Beresi V., Quintane J., DeI Pozo H.: Leucemia linfoblástica aguda del nifio. Rev. Chil. Pediatr. 50: 12, 1979.

21 Advis P., Burgos O., Diaz R., Gonzúlez G., Olivares, Pinto $F .:$ Compromiso dẹl sistema nervioso central en leucemia linfoblástica. Jomada Científica Anuał $1^{\circ}-2 /$ Dic./83, Santiago, Soc. Chil. Hematología, p 8.
22 Robinson LL. Sather H. Coccta PF, Nesbir ME. Hommond GD: Assesment of the interrelationshjp of prognostic factors in childhood acute lymphoblastic leukemia. A report from Childrens Cancer Study Group. An J. Pediatr. Hematol. Oncol. 2: $5 \mathrm{~m} \cdot 14$, 1980 .

23 Simone $J V$ : Leukemia remission and survival. Larcet (letter) 2: 531,1981 .

24 Kay HEM, Peto $J_{\text {, }}$ Rankin $A$.: The treatment of acute lymphoblastic leukemia (ALL) in childhood, Ukall IIl. Med. Pediatr. Oncol. 10: 501, 1982.

25 Miller DR, Leikin S. Albo V., Sather H., Karon W., Hammond $D$.: Prognostic factors and therapy in acute lymphoblastic leukemia of childhood: CCG-141. A report from Childrens Cancer Study Group. Cancer 51: $1041,1983$.

${ }^{26}$ Komp DM, Fernández CH, Falleta JM, Ragab AH. Humphrey GB, Pullen J., Moon T., Shuster J.: CNS prophylaxis in acute lymphoblastic leukemia. Cances 50: 1031, 1982.

27 Dahl GV, Simone JV, Hustu HO, Mason C.: Preventive central nervous system irradiation in children with acute nonlymphocytic leukemia. Cancer 42: $2187,1978$.

28 Choi SI, Simone IV: Acute nonlymphocytic leukemia in 171 children. Med. Pediatr. Oncol, 2: $119,1976$.

29 Weinstein HJ, Mayer RJ, Rosenthal DS, Camitra BM, Coral FS, Nathan $D G$, Frei E.: Treatment of acute myelogenous leukemia in children and adults. $N$. Engl, J. Med. 303: 473, 1980 .

${ }^{30}$ Madanat $F F$, Sultivan MP: Improved survival in young children with acute granulocytic leukemia treated with combination therapy using cyclophosphamide, oncovin, cytosine arabinoside and prednisone. Cancer $44: 819,1979$

31 Pochedly C.: Neutologic manifestations in acute leukemia. II. lnvolvement of cranial nerves and hypothalamus. N.Y. State J. Med. 75:715, 1975.

32 Kay HEM, Developement of CNS leukemia in acute myeloid leukemia in childhood. Arch. Dis. Child. 51: $73,1976$.

33 Thigpen JT, Morrison FS, Smith DL, Maier FG.: Diagnostic considerations in CNS leukemia. South. Med. J. 69: 76, 1976.

34 Pochedly $C$. Neurotoxicity due to CNS therapy for leukemia. Med. Pediatt. Oncol. 3: 101, 1977.

35 Simone JV, Aur RJ, Hustu HO, Verzose MS, Pinkel $D$.: Combined modality theraphy of acute lymphocy tic leukemia. Cancer 35: 25, 1975

${ }^{36}$ Mauer AM, Simone JV: The current status of the treatment of childhood acute lymphoblastic leukemia. Cancer Treat. Rev. 3: 17, 1976.

37 Foon $X A$, Gole RP; Controversies in the therapy of acute myelogenous teukemia. Am. J. Med. 72: 963, 1982 .

38 Wolff $L$. , Zighelboim $J$, Gole RP.: Paraplegia following intrathecal cytosine arabinoside. Cancer 43: 83, 1979.

39 Eden $O B$, Goldie W., Wood T, Etcubanas E.: Seizures following intrathecal cytosine arabinoside in young children with acute lymphoblastic leukemia. Cancer 42: 53, 1978.

40 Haghbin M., Murphy L., Tan ChC, Clarkson BD, Thaler HT, Passe S, Burchenal J.: A long-term clinical follow-up of children with acute lymphoblastic leukemia treated with intensive chemotherapy regimens. Cancer 46: 241, 1980.

41 GOPECH: Principales enfermedades oncológicas infantiles. Normas terapéuticas y de estudio, Santiago de Chile, 1982. 
42 Sallan SE, Weinstein HJ, Nathan DG: The childhood leukemias. J. Pediatr. 99: 676, $19 \mathrm{B1}$.

${ }^{43}$ Ch'ien LT, Aur RJ, Verzosa MS, Coburn TP, Goff $J R$, Hustu HO, Price RA, Seifert J., Simone JV: Progression of methotrexate-induced leukoencephalopathy in children with leukemia. Med. Pediatr. Oncol 9: 133, 1981.

44 Allen $J C$ : The effects of cancer therapy on the nervous system. J. Pediatr. 93: 903, 1978.

45 Addiego $J E$, Ridway $D$, Bleyer $W A$ : The acute management of intrathecal methotrexate overdose: pharmacologic rationale and guidelines. J. Pediatr. 90; 825, 1981.

46 Bertino JR: "Rescue" techniques in cancer chemotherapy: use of leucovorin and other rescue agents after methotrexate treatment. Semin. Oncol 4: 203, 1977 .

47 Bradsrock, K.F.; Papageorgiou, E.S.; Janossy, G:: Diagnosis of meningeal involvement in patients with acute lymphoblastic leukemia: Immunofluorescence for terminal transferase. Cancer 47: 2478, 1981.

4 a Casper, J.T.. Louer, JS.; Kirchner. PA.: Gottschall, $J_{. L}$. Camitta, B.M.: Evaluation of cerebrospinal fluid mononuclear cells obtained from children with acute lymphocytic leukemia: advantages of combining cy tomorphology and terminal deoxynucleotidyl transferase. Am. J. Clin. Pathol 80: 666, 1983.

49 Sullivan, M.P.: Chen, T.: Dyment, P.G.: Hyizdaia, E..
Stetiber, C.P.: Equivalence of intrathecal chemotherapy and radiotherapy as central nervous system prophylaxis in children with acute lymphatic leukemia: A Pediatric Oncology Group Study. Blood 60: 948,1982 .

50 Kende, G.; El-Najjar, K.; Ben-Bassat, I.; Neuman, Y.: Ballin. A.; Ramot, B.: Result of treatment of high risk childhood acute lymphoblstic leukemia. Med, Pediatr. Oncol, 11: 49, 1983.

51 Sackmann, F.; Suarch, E.; Pavlosky, S.; EppingerHelft, $M_{\text {.; }}$ Braier, J.; Vergara, B.: Garay, G.: Xvicala. R.; Divito, J.M.; Failace, R.; Dibar, E.; Jiménez, E.: Comparison of central nervous system prophylaxis with cranial irjadiation and intrathecal methotrexate versus intrathecal methotrexate alone in acute lymphoblastic leukemia. Blood 62: 241, 1983.

$\$ 2$ Moss, H.A.; Nannis, E.D.; Poplack, D.G.: The effects of prophylactic treatment of the central nervous system on the intellectual functioning of children with acute lymphocytic leukemia. Am. J. Med. $71: 47,1981$.

${ }^{53}$ Inati, A.: Sallan, S.E.; Cassady, J.R.; HitchoockBryan, S.; Clavell, L.A.; Belli, J.A.; Sollee, N.: Elficacy and morbidity of central nervous system "prophylaxis" in childhood acute lymphoblastic leukemia: Eight years' experience with cranial irradiation and intrathecal methotrexate. Blood 61: 297 , 1983. 